\title{
Yếu tố ảnh hưởng đến hiệu quả giảng dạy của giảng viên
}

\author{
Nguyễn Thị Phương Thảo ${ }^{1}$, Võ Văn Việt ${ }^{2, *}$ \\ ${ }^{I}$ Truờng Đại học Công nghệ Thông tin, Đại học Quốc gia TPHCM \\ ${ }^{2}$ Truờng Đại học Nông Lâm TPHCM \\ Nhận ngày 03 tháng 4 năm 2017 \\ Chỉnh sửa ngày 28 tháng 4 năm 2017; Chấp nhận đăng ngày 20 tháng 6 năm 2017
}

\begin{abstract}
Tóm tắt: Hiệu quả giảng dạy của giảng viên được xem là yếu tố quan trọng quyết định chất lượng giáo dục đại học. Một khi giảng viên thỏa mãn, yêu thích với công việc của mình, họ sẵn sàng phấn đấu hết mình để công việc ngày một tốt hơn, điều này góp phần vào sự thành công của một trường đại học, đồng thời xây dựng được nguồn nhân lực có trình độ phục vụ cho đất nước. Mục tiêu của nghiên cứu này nhằm xác định các yếu tố ảnh hưởng đến hiệu quả giảng dạy của giảng viên. Trên cơ sở thực trạng được khảo sát, xử lý, phân tích kết quả nghiên cứu cho thấy các yểu tố: sự phản hồi và kết quả của sinh viên; Đồng nghiệp; Cơ sở vật chất; Lương, thưởng và phụ cấp lần lượt ảnh hưởng đến hiệu quả giảng dạy của giảng viên.
\end{abstract}

Tù khóa: Hiệu quả giảng dạy, giảng viên, chất lượng đào tạo.

\section{1. Đặt vấn đề}

Hướng tới sự phát triển, mỗi quốc gia trên thế giới đều có những chiến lược riêng của mình, song không một quốc gia nào trong sự phát triển lại không có sự đầu tư cho giáo dục. Hiệu quả giảng dạy của giảng viên được xem là yếu tố quan trọng quyết định chất lượng giáo dục. Do đó, tìm hiểu về các yếu tố ảnh hưởng đến hiệu quả giảng dạy của giảng viên tại nơi làm việc rất quan trọng cho sự thành công của một trường đại học. Giảng viên giảng dạy có hiệu quả giúp cơ sở nâng cao chất lượng đào tạo, góp phần xây dựng nguồn nhân lực có trình độ phục vụ đất nước. Nhiều nghiên cứu cho thấy hiệu quả giảng dạy quyết định đến kết quả học tập của sinh viên và được xem là yếu tố quyết định quan trọng nhất trong việc nâng cao thành tích học tập của sinh viên [1-3].

\footnotetext{
*Tác giả liên hệ. ĐT.: 84-4-908849631.

Email: vietvovan@yahoo.com

https://doi.org/10.25073/2588-1159/vnuer.4066
}

Bên cạnh đó, các nhà nghiên cứu đã theo dõi chặt chẽ thành tích của người học và xác định hiệu quả của giáo viên là kết quả lâu dài đối với sự thành công của người học $[4,5]$. Vai trò của giáo viên không đơn giản chỉ đứng trước lớp học và giảng dạy mà còn hỗ trợ kết nối người học, từ đó người học học tập tốt hơn thông qua quá trình giáo dục trong môi trường học tập tích hợp. Nói cách khác, giáo viên giảng dạy hiệu quả không chỉ đơn thuần là dạy học mà còn kết hợp nhiều nhiệm vụ trong một tiết dạy để đảm bảo tất cả người học đều nhận được nền giáo dục có chất lượng.

Vậy yếu tố nào tác động đến việc thu hút nguồn nhân lực, tác động đến hiệu quả công việc hay hiệu quả giảng dạy. Trả lời cho những câu hỏi này, nhiều nhà nghiên cứu trên thế giới đã nghiên cứu về sự thỏa mãn ảnh hưởng đển hiệu quả công việc như: Spector, Luddy...[6, 7]. Các yếu tố quyết định sự hài lòng công việc đã được nghiên cứu rộng rãi ở nhiều nước phát triển trên thế giới [8]. Đồng thời nghiên cứu của 
Ellickson và Logsdon năm 2002 cho rằng sự hài lòng trong công việc là mức độ nhân viên yêu thích công việc của họ, đó là thái độ dựa trên nhận thức của người nhân viên (tích cực hay tiêu cực) về công việc hoặc môi trường làm việc của họ. Nói đơn giản hơn, môi trường làm việc càng đáp ứng các nhu cầu, giá trị và tính cách của người lao động thì mức độ thỏa mãn công việc càng cao [9]. Nghiên cứu này được thực hiện nhằm mục tiêu: (1) xác định các yếu tố ảnh hưởng đến hiệu quả giảng dạy của giảng viên; (2) đề xuất giải pháp nhằm nâng cao hiệu quả giảng dạy của giảng viên.

Các giả thuyết nghiên cứu:

$\mathrm{H}_{1}$ : Bản chất công việc ảnh hưởng đến Hiệu quả giảng dạy của giảng viên

$\mathrm{H}_{2}$ : Lương, thưởng và phụ cấp ảnh hưởng đến Hiệu quả giảng dạy của giảng viên

$\mathrm{H}_{3}$ : Quan hệ đồng nghiệp ảnh hưởng đến Hiệu quả giảng dạy của giảng viên

$\mathrm{H}_{4}$ : Quản lý, lãnh đạo ảnh hưởng đến Hiệu quả giảng dạy của giảng viên

$\mathrm{H}_{5}$ : Cơ hội đào tạo và thăng tiến ảnh hường đến Hiệu quả giảng dạy của giảng viên

$\mathrm{H}_{6}$ : Cơ sở vật chất ảnh hưởng đến Hiệu quả giảng dạy của giảng viên

$\mathrm{H}_{7}$ : Sự phản hồi và kết quả của sinh viên ảnh hưởng đến Hiệu quả giảng dạy của giảng viên

\section{Phương pháp nghiên cứu-thang đo}

Để đạt được mục tiêu nghiên cứu đề ra và làm sáng tỏ các giả thuyết nghiên cứu, tiếp cận nghiên cứu định lượng đã được vận dụng. Công cụ chính để thu thập dữ liệu là bảng câu hỏi được thiết kế sẵn. Bảng câu hỏi gồm 2 phần, phần một để thu thập các thông tin về nhân khẩu học và phần hai là các phát biểu để đo lường các yếu tố ảnh hưởng đến hiệu quả giảng dạy với thang đo likert 5 cấp độ.

Kích thước của mẫu là 176 , mẫu nghiên cứu được thu thập bằng phương pháp thuận tiện trên tổng thể là giảng viên của trường Đại học Công nghệ thông tin, Đại học Quốc gia Thành phố Hồ Chí Minh.

Dũ liệu sau khi được thu thập sẽ tiến hành mã hóa, nhập số liệu, làm sạch với phần mềm SPSS version 23.0.

Độ tin cậy của thang đo được đánh giá bằng phương pháp hệ số tin cậy Cronbach's Alpha. Sử dụng phương pháp hệ số tin cậy Cronbach's Alpha trước khi phân tích nhân tố EFA để loại các biến không phù hợp vì các biến rác này có thể tạo ra các yếu tố giả [10]. Hệ số tin cậy Cronbach's Alpha chỉ cho biết các đo lường có liên kết với nhau hay không; nhưng không cho biết biến quan sát nào cần bỏ đi và biến quan sát nào cần giữ lại. Khi đó, việc tính toán hệ số tương quan giữa biến tổng sẽ giúp loại ra những biến quan sát nào không đóng góp nhiều cho sự mô tả của khái niệm cần đo [11]. Các tiêu chí được sử dụng khi thực hiện đánh giá độ tin cậy thang đo:

Loại các biến quan sát có hệ số tương quan biến tổng nhỏ (nhỏ hơn 0,3 ); tiêu chuẩn chọn thang đo khi có độ tin cậy Alpha lớn hơn 0,6. Hệ số tương quan biến tổng thể là hệ số tương quan của một biến với điểm trung bình của các biến khác trong cùng một thang đo, do đó hệ số này càng cao, sự tương quan của các biến với các biến khác trong nhóm càng cao. Theo Nunally \& Burnstein (1994) thì các biến có hệ số tương quan biến tổng nhỏ hơn 0,3 được xem là biến rác và đương nhiên là loại bỏ khỏi thang đo [12].

Phân tích nhân tố EFA dùng để kiểm định giá trị khái niệm của thang đo. Các biến có trọng số thấp (nhỏ hơn 0,4 ) sẽ bị loại và thang đo chỉ được chấp nhận khi tổng phương sai trích lớn hơn 0,5 .

Và cuối cùng là phân tích hồi quy tuyến tính bộ (multiple regression analysis) để kiểm định mô hình nghiên cứu và giả thuyết nghiên cứu.

\section{Kết quả nghiên cứu}

\subsection{Mô tả mẫu nghiên cúu}

Theo bảng 1 ta thấy do đặc thù đào tạo về Công nghệ Thông tin nên số lượng giảng viên nam chiếm khá cao. Tổng số giảng viên nam được khảo sát là 107 người, chiếm tỉ lệ $60,8 \%$; 
tổng số giảng viên nữ khảo sát là 69 người, chiếm tỉ lệ 39,2\%. Trường Đại học Công nghệ Thông tin mới được thành lập từ năm 2006 nên độ tuổi trung bình của giảng viên còn khá trẻ. Đối tượng khảo sát dưới 30 tuổi là 64 người, chiếm tỉ lệ 36,4\%; đối tượng khảo sát từ 30 - 40 tuổi là 87 người, chiếm tỉ lệ 49,4\%; đối tượng khảo sát từ 41 tuổi trở lên là 25 người, chiếm tỉ lệ $14,2 \%$.

Bảng 1. Đặc điểm nhân khẩu học của mẫu nghiên cứu

\begin{tabular}{|c|c|c|c|}
\hline Tiêu chí & & Số lượng & Tỉ lệ \% \\
\hline \multirow{2}{*}{ Giới tính } & Nam & 107 & 60,8 \\
\hline & Nữ & 69 & 39,2 \\
\hline \multirow{4}{*}{ Độ tuổi } & Dưới 30 tuổi & 64 & 36,4 \\
\hline & Từ 30 tuổi đến 40 tuổi & 87 & 49,4 \\
\hline & Từ 41 tuổi trở lên & 25 & 14,2 \\
\hline & Đại học & 21 & 11,9 \\
\hline \multirow{2}{*}{ Trình độ } & Thạc sĩ & 127 & 72,2 \\
\hline & Tiến sĩ & 23 & 13,1 \\
\hline \multirow{6}{*}{ Thâm niên công tác } & Phó giáo sư & 5 & 2,8 \\
\hline & Dưới 1 năm & 15 & 8,5 \\
\hline & Từ 1 đến 2 năm & 22 & 12,5 \\
\hline & Từ 3 đến 5 năm & 36 & 20,5 \\
\hline & Trên 5 năm & 103 & 58,5 \\
\hline & Tổng & 176 & 100 \\
\hline
\end{tabular}

Là Trường đào tạo trình độ đại học trở lên nên yêu cầu tối thiểu của giảng viên giảng dạy lý thuyết tối thiểu phải là thạc sĩ vì vậy số lượng giảng viên có học vị thạc sĩ chiếm đa số gồm 127 người, chiếm 72,2\%; số lượng giảng viên có học vị đại học chiếm tỉ lệ thấp vì chỉ được giảng dạy các môn thực hành hoặc là trợ giảng gồm 21 người, chiếm tỉ lệ $11,9 \%$; số lượng giảng viên có học vị tiến sĩ gồm 23 người, chiếm tỉ lệ $13,1 \%$; giảng viên có học vị trên tiến sĩ gồm 5 người, chiếm tî̉ lệ $2,8 \%$.

Thâm niên công tác của đa số giảng viên công tác trên 5 năm chiếm tối đa gồm 103 người, chiếm tỉ lệ 58,5\%; Trường đã tuyển gần đủ số lượng biên chế quy định nên việc tuyển thêm giảng viên rất hạn chế vì vậy số lượng giảng viên công tác dưới 1 năm chiếm thấp nhất gồm 15 người, chiếm tỉ lệ $8,5 \%$; số lượng giảng viên công tác từ 3 đến 5 năm gồm 36 người, chiếm tỉ lệ $20,5 \%$; số lượng giảng viên công tác từ 1 đến 2 năm gồm 22 người, chiếm tỉ lệ 12,5\%.

\subsection{Kiểm định mô hình và giả thuyết nghiên cứu}

Mô hình lý thuyết đề xuất gồm có 7 thành phần: (i) Bản chất công việc; (ii) Lương, thưởng và phụ cấp; (iii) Đồng nghiệp; (iv) Quản lý, lãnh đạo; (v) Cơ hội đào tạo và thăng tiến; (vi) Cơ sở vật chất; (vii) Sự phản hồi và kết quả học tập của sinh viên và Hiệu quả giảng dạy của giảng viên. Trong đó, Hiệu quả giảng dạy của giảng viên là thành phần phụ thuộc, 7 thành phần còn lại là những thành phần độc lập và được giả định là các yếu tố tác động đến Hiệu quả giảng dạy của giảng viên.

Hình dạng phương trình:

$$
Y=\beta_{1} X_{1}+\beta_{2} X_{2}+\beta_{3} X_{3}+\beta_{4} X_{4}+
$$$$
\beta{ }_{5} X_{5}+\beta{ }_{6} X_{6}+\beta{ }_{7} X_{7}
$$

Tiến hành phân tích hồi qui để xác định cụ thể trọng số của từng thành phần tác động đến Hiệu quả giảng dạy của giảng viên. Giá trị của các yếu tố được dùng để chạy hồi qui là giá trị tổng của các biến quan sát đã được kiểm định. Phân tích hồi qui được thực hiện bằng phương 
pháp hôi qui tổng thể các biến với phần mềm SPSS version 23.0.

Kết quả kiểm định mô hình hồi quy giữa các yếu tố tác dộng đến hiệu quả giảng dạy được thể hiện qua hệ thống các bảng sau:

Trị số $\mathrm{R}$ có giá trị 0,868 cho thấy mối quan hệ giữa các biến trong mô hình có mối tương quan rất chặt chẽ. Báo cáo kết quả hồi qui của mô hình cho thấy giá trị $\mathrm{R} 2$ ( $\mathrm{R}$ Square) bằng 0,753 , điều này nói lên độ thích hợp của mô hình là 75,30\% hay nói cách khác là 75,30\% sự biến thiên của biến Hiệu quả giảng dạy được giải thích bởi 7 thành phần. Giá trị R điều chỉnh (Adjusted R Square) phản ánh chính xác hơn sự phù hợp của mô hình đối với tổng thể, ta có giá trị $\mathrm{R}$ điều chỉnh bằng 0,743 (hay $74,30 \%$ ) có nghĩa tồn tại mô hình hồi qui tuyến tính giữa hiệu quả giảng dạy và 7 thành phần trong yếu tố ảnh hưởng đế hiệu quả giảng dạy.

Bảng 2. Kết quả hồi qui của mô hình

\begin{tabular}{cccccc}
\hline Mô hình & $\mathrm{R}$ & $\mathrm{R}^{2}$ & $\mathrm{R}^{2}$ hiệu chỉnh & Sai số chuấn của ước lượng & Durbin-Watson \\
\hline 1 & $0,868^{\mathrm{a}}$ & 0,753 & 0,743 & 1,45555 & 1,997 \\
\hline
\end{tabular}

a. Các yếu tố dự báo: (Hằng số), X7, X2, X4, X5, X6, X1, X3

b. Biến phụ thuộc: $Y$

Hệ số Durbin Watson dùng để kiểm định tương quan chuỗi bậc nhất cho thấy mô hình không vi phạm khi sử dụng phương pháp hồi quy bội vì giá trị $\mathrm{DW}$ đạt được là 1,997 (nằm trong khoảng từ 1 đến 3 ) và chấp nhận giả thuyết không có sự tương quan chuỗi bậc nhất trong mô hình. Như vậy, mô hình hồi quy bội thỏa các điều kiện đánh giá và kiểm định độ phù hợp cho việc rút ra các kết quả nghiên cứu.

Phân tích phương sai ANOVA cho thấy trị số $\mathrm{F}$ có mức ý nghĩa Sig. $=0,000$ (nhỏ hơn
$0,05)$, có nghĩa là mô hình hồi qui phù hợp với sữ liệu thu thập được và các biến đưa vào đều có ý nghĩa trong thống kê với mức ý nghĩa $5 \%$. Thống kê giá trị $\mathrm{F}=73,096$ được dùng để kiểm định giả thuyết $\mathrm{H}_{0}$, mối quan hệ tuyến tính là rất có ý nghĩa với Sig. $<0,05$. Ta có thể bác bỏ giả thuyết $\mathrm{H}_{0}$ cho rằng hệ số góc của 7 thành phần trong yếu tố ảnh hưởng đến hiệu quả giảng dạy bằng 0 . Như vậy, các biến độc lập trong mô hình có quan hệ đối với biến phụ thuộc Hiệu quả giảng dạy.

Bảng 3. Phân tích phương sai ANOVA

\begin{tabular}{ccccccc}
\hline Mô hình & $\begin{array}{c}\text { Tổng } \\
\text { bình phương }\end{array}$ & $\begin{array}{c}\text { Bậc } \\
\text { tự do }\end{array}$ & $\begin{array}{c}\text { Trung bình bình } \\
\text { phương }\end{array}$ & F & $\begin{array}{c}\text { Mức ý } \\
\text { nghĩa }\end{array}$ \\
\hline \multirow{2}{*}{1} & Hồi qui & 1084,047 & 7 & 154,864 & 73,096 & $0,000^{b}$ \\
& Số dư & 355,930 & 168 & 2,119 & & \\
& Tổng & 1439,977 & 175 & & & \\
\hline
\end{tabular}

a. Biến phụ thuộc: Y

b. Các yếu tố dự báo: (Hằng số), X7, X2, X4, X5, X6, X1, X3

Kết quả phân tích các hệ số hồi qui trong mô hình cho thấy, mức ý nghĩa của các thành phần X1, X3, X4, X7 Sig. $=0,000<0,05$; biến X2, X5, X6 có Sig. lần lượt là $0,192,0,554$, 0,327 nên các biến X2, X5, X6 bị loại khỏi mô hình. Các biến độc lập (X1, X3, X4, X7) có Sig. $<0,05$ vì vậy có tác động đến hiệu quả giảng dạy của giảng viên. Bốn thành phần này đều có ý nghĩa trong mô hình và tác động cùng chiều đến hiệu quả giảng dạy của giảng viên, do các hệ số hồi qui đều mang dấu dương.

Đại lượng chuẩn đoán hiện tượng đa cộng tuyến với hệ số phóng đại phương sai VIF (Variance Inflation Factor) đều $<2$, thể hiện tính đa cộng tuyến của các biến độc lập là không đáng kể và các biến độc lập trong mô hình chấp nhận được. 
Bảng 4. Các hệ số hồi qui trong mô hình

\begin{tabular}{|c|c|c|c|c|c|c|c|c|}
\hline & \multirow{2}{*}{ Mô hình } & \multicolumn{2}{|c|}{$\begin{array}{c}\text { Các hệ số } \\
\text { chưa chuẩn hóa }\end{array}$} & \multirow{2}{*}{$\begin{array}{c}\text { Các hệ số } \\
\text { chuẩn hóa } \\
\text { Beta }\end{array}$} & \multirow[t]{2}{*}{$\mathrm{t}$} & \multirow{2}{*}{$\begin{array}{l}\text { Mức ý } \\
\text { nghĩa }\end{array}$} & \multicolumn{2}{|c|}{ Thống kê Collinearity } \\
\hline & & $\mathrm{B}$ & Sai số chuấn & & & & Dung sai & VIF \\
\hline \multirow[t]{8}{*}{1} & (Hằng số) & 16,194 & 0,983 & & 16,471 & 0,000 & & \\
\hline & $\mathrm{X} 1$ & 0,112 & 0,042 & 0,132 & 2,655 & 0,009 & 0,595 & 1,681 \\
\hline & $\mathrm{X} 2$ & $-0,037$ & 0,029 & $-0,053$ & $-1,311$ & 0,192 & 0,911 & 1,098 \\
\hline & $\mathrm{X} 3$ & 0,597 & 0,055 & 0,583 & 10,848 & 0,000 & 0,510 & 1,961 \\
\hline & $\mathrm{X} 4$ & 0,287 & 0,049 & 0,263 & 5,868 & 0,000 & 0,730 & 1,370 \\
\hline & X5 & $-\mathbf{0 , 0 4 0}$ & 0,068 & $-0,030$ & $-0,594$ & 0,554 & 0,559 & 1,790 \\
\hline & X6 & $-0,048$ & 0,048 & $-0,044$ & $-0,983$ & $\mathbf{0 , 3 2 7}$ & 0,724 & 1,382 \\
\hline & $\mathrm{X} 7$ & 0,222 & 0,063 & 0,172 & 3,494 & 0,001 & 0,605 & 1,653 \\
\hline
\end{tabular}

a. Biến phụ thuộc: Y

Các biến đều đạt được tiêu chuẩn chấp nhận (Tolerance $>0,0001$ ).

Giá trị hồi qui chuẩn của các biến độc lập trong mô hình có giá trị báo cáo lần lượt: Lương, thưởng và phụ cấp là 0,132 ; Sự phản hồi và kết quả của sinh viên là 0,583 ; Đồng nghiệp là 0,263 ; Cơ sở vật chất là 0,172 .

Qua kết quả phân tích hồi qui ta có mô hình:

$\mathrm{Y}=0,583 \mathrm{X}_{3}+0,263 \mathrm{X}_{4}+0,172 \mathrm{X}_{7}+$ $0,132 \mathrm{X}_{1}$

Mô hình trên giả thích được 74,30\% sự thay đổi của biến $\mathrm{Y}$ là do các biến độc lập trong mô hình tạo ra, còn lại $25,70 \%$ biến thiên được giải thích bởi các biến khác nằm ngoài mô hình.

Mô hình cho thấy các biến độc lập đều ảnh hưởng thuận chiều đến kết quả học tập của sinh viên ở độ tin cậy 95\%. Qua phương trình hồi qui cho thấy, nếu giữ nguyên các biến độc lập còn lại không đổi thì khi điểm đánh giá về Lương, thưởng và phụ cấp tăng lên 1 thì hiệu quả giảng dạy của giảng viên tăng trung bình lên 0,132 điểm. Tương tự, khi điểm đánh giá về Sự phản hồi và kết quả của sinh viên tăng lên 1 điểm thì hiệu quả giảng dạy của giảng viên tăng lên trung bình 0,583 điểm; khi điểm đánh giá về Đồng nghiệp tăng lên 1 điểm thì hiệu quả giảng dạy của giảng viên tăng lên trung bình 0,263 điểm; khi điểm đánh giá về Cơ sở vật chất tăng lên 1 điểm thì hiệu quả giảng dạy của giảng viên tăng lên trung bình 0,172 điểm.

Tổng hợp kết quả kiểm định mô hình hồi qui với 7 biến độc lập và 1 biến phụ thuộc.

\begin{tabular}{|c|c|}
\hline Giả thuyết & Kết quả kiểm định \\
\hline $\mathrm{H}_{1}$ : Bản chất công việc ảnh hưởng đến Hiệu quả giảng dạy của giảng viên & Không chấp nhận \\
\hline $\begin{array}{l}\mathrm{H}_{2} \text { : Lương, thưởng và phụ cấp ảnh hưởng đến Hiệu quả giảng dạy của giảng } \\
\text { viên }\end{array}$ & Chấp nhận \\
\hline $\mathrm{H}_{3}$ : Quan hệ đồng nghiệp ảnh hưởng đến Hiệu quả giảng dạy của giảng viên & Chấp nhận \\
\hline $\mathrm{H}_{4}$ : Quản lý, lãnh đạo ảnh hưởng đến Hiệu quả giảng dạy của giảng viên & Không chấp nhận \\
\hline $\begin{array}{l}\mathrm{H}_{5}: \text { Co hội đào tạo và thăng tiến ảnh hưởng đền Hiệu quả giảng dạy của giảng } \\
\text { viên }\end{array}$ & Không chấp nhận \\
\hline $\mathrm{H}_{6}$ : Cơ sở vật chất ảnh hưởng đến Hiệu quả giảng dạy của giảng viên & Chấp nhận \\
\hline $\begin{array}{l}\mathrm{H}_{7} \text { : Sự phản hồi và kết quả của sinh viên ảnh hưởng đến Hiệu quả giảng dạy } \\
\text { của giảng viên }\end{array}$ & Chấp nhận \\
\hline
\end{tabular}

Qua bảng trên chúng ta thấy các giả thuyết $\mathrm{H} 2, \mathrm{H} 3, \mathrm{H} 6$ và $\mathrm{H} 7$ đều được chấp nhận, các thành phần Lương, thưởng và phụ cấp; Quan hệ đồng nghiệp; Cơ sở vật chất; Sự phản hồi và kết quả của sinh viên có ảnh hưởng đến hiệu quả giảng dạy của giảng viên, khi tăng những yếu tố này sẽ làm gia tăng hiệu quả giảng dạy của giảng viên. Vì vậy, Trường phải nổ lực cải tiến những yếu tố này để nâng cao hiệu quả giảng dạy. 
Đồng thời ta thấy yếu tố Bản chất công việc không được chấp nhận có thể nói rằng giảng yêu thích công việc hiện tại nên yếu tố này ảnh hưởng không nhiều đến đa số giảng viên tại Trường. Cũng chính vì yêu thích công việc nên việc lãnh đạo có quan tâm hay không, giảng viên có cơ hội được đào tạo và thăng tiến hay không cũng không quan trọng với giảng viên. Xem kết quả trên hai yếu tố này cũng không được chấp nhận vì vậy cũng không ảnh hưởng nhiều đến hiệu quả giảng dạy của giảng viên.

Từ những phân tích trên ta có thể kết luận mô hình lý thuyết thích hợp với dữ liệu nghiên cứu và các giả thuyết nghiên cứu được chấp nhận (giả thuyết $\mathrm{H} 2, \mathrm{H} 3, \mathrm{H} 6$ và $\mathrm{H} 7$ ). Kết quả kiểm định mô hình lý thuyết được minh họa qua hình sau.

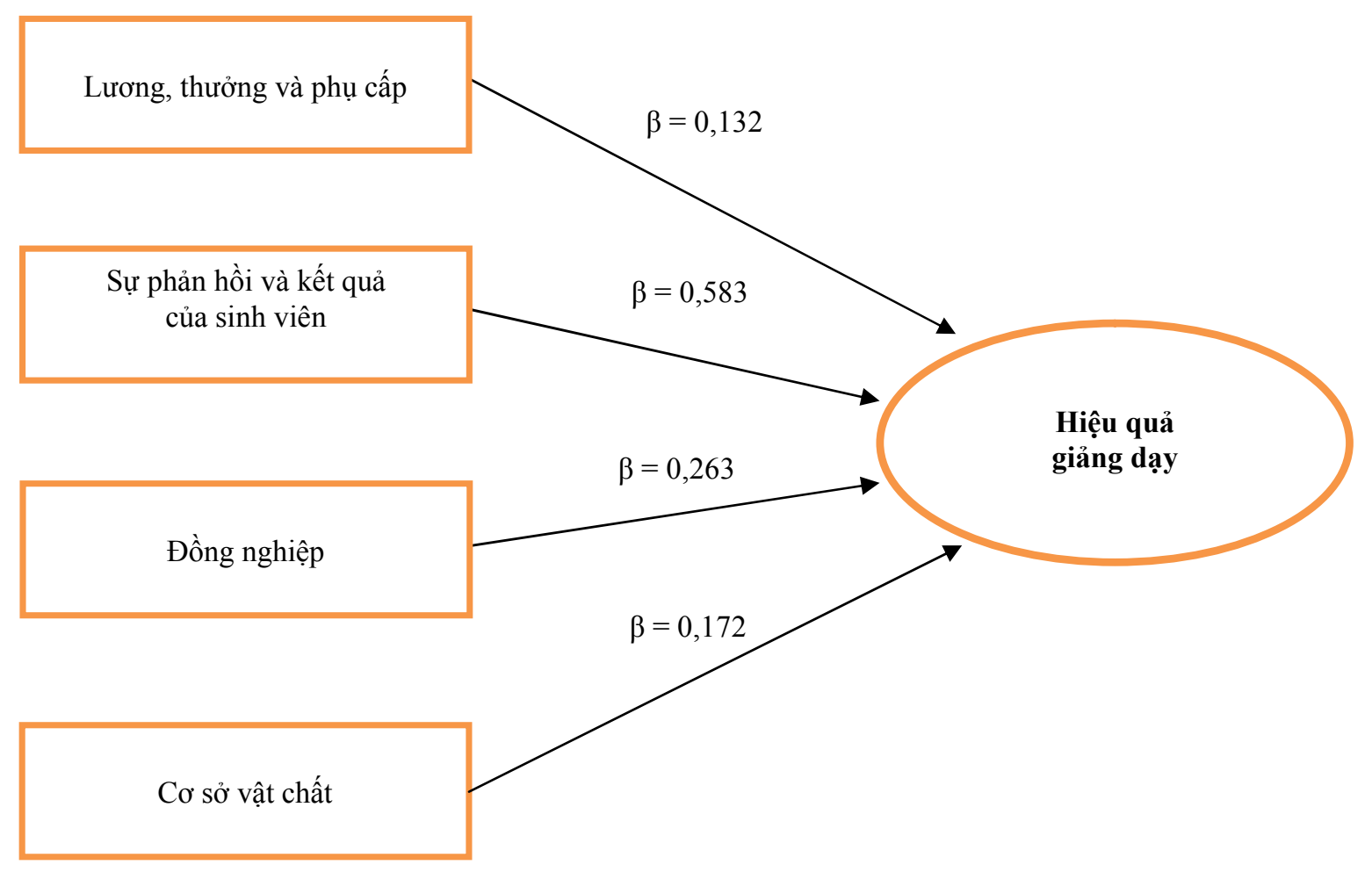

Hình 1. Kết quả kiểm định mô hình lý thuyết.

Qua hình 1 cho ta thấy được tầm quan trọng của các thành phần phụ thuộc vào giá trị tuyệt đối của hệ số hồi qui đã chuẩn hóa. Thành phần nào có giá trị tuyệt đối càng lớn thì ảnh hưởng đến hiệu quả giảng dạy càng nhiều. Do đó, có thể nói rằng hiệu quả giảng dạy chịu ảnh hưởng nhiều nhất từ thành phần Sự phản hồi và kết quả của sinh viên (Beta $=0,583)$; quan trọng thứ hai là thành phần Đồng nghiệp (Beta = $0,263)$; quan trọng thứ ba là thành phần $\mathrm{Cơ}$ sở vật chất $($ Beta $=0,172)$; và cuối cùng là thành phần Lương, thưởng và phụ cấp $($ Beta $=0,132)$.

\section{Kết luận và khuyến nghị}

Mục tiêu chính của nghiên cứu là xác định các yếu tố ảnh hưởng đến hiệu quả giảng dạy của giảng viên tại trường đại học. Tác giả đã xây dựng và đánh giá các thang đo lường các thành phần có ảnh hưởng đến hiệu quả giảng dạy. Để khẳng định sự tác động của các thành phần này vào hiệu quả giảng dạy của giảng viên, một mô hình lý thuyết được xây dựng và kiểm định.

Các thang đo lường các thành phần ảnh hưởng đến hiệu quả giảng dạy của giảng viên 
sau khi đề xuất và bổ sung đều đạt được độ tin cậy và giá trị cho phép. Theo mô hình nghiên cứu lý thuyết, có 7 yếu tố ảnh hưởng đến hiệu quả giảng dạy của giảng viên bao gồm: (1) Bản chất công việc; (2) Lương, thưởng và phụ cấp; (3) Quan hệ đồng nghiệp; (4) Quản lý, lãnh đạo; (5) Cơ hội đào tạo và thăng tiến; (6) Cơ sở vật chất; (7) Sự phản hồi và kết quả của sinh viên và thang đo hiệu quả giảng dạy của giảng viên. Kết quả kiểm định mô hình lý thuyết cho thấy chỉ có 04 thành phần ảnh hưởng đến hiệu quả giảng dạy của giảng viên. Trong đó thành phần tác động mạnh nhất đến hiệu quả giảng dạy của giảng viên là thành phần Sự phản hồi và kết quả của sinh viên $($ Beta $=0,583)$; thứ hai là thành phần Quan hệ đồng nghiệp (Beta $=$ $0,239)$; thứ ba là thành phần Cơ sở vật chất $($ Beta $=0,172)$; và cuối cùng là thành phần Lương, thưởng và phụ cấp $($ Beta $=0,132)$.

\section{Khuyến nghị}

Kết quả nghiên cứu cho thấy sư phản hồi và kết quả của sinh viên là yếu tố ảnh hưởng nhiều nhất đến hiệu quả giảng dạy của giảng viên. Trong đó các nhóm nhân tố về thái độ học tập, sự sáng tạo của sinh viên là nguồn cảm hứng và nhiệt huyết cho giảng viên; sinh viên có hứng thú với môn học và trao đồi thường xuyên với giảng viên cũng ảnh hưởng không nhỏ đến hiệu quả giảng dạy. Vì vậy, cần cải tiến phương pháp giảng dạy để lôi cuốn được sự chú ý của sinh viên. Tăng cường đặt câu hỏi và khuyến khích người học đưa ra câu hỏi; ví dụ như giảng viên có thể tạo sự tham gia tích cực của học viên bằng cách đưa ra nhiều câu hỏi buộc sinh viên phải tích cực suy nghĩ tìm cách trả lời và khuyến khích sinh viên đặt câu hỏi để có thể biết được sinh viên hiểu bài ở mức độ nào.

Bên cạnh đó, Nhà trường nên tổ chức các buổi tập huấn ngoài giờ lên lớp nhiều hơn, giúp sinh viên có thể trao đổi, học hỏi lẫn nhau, cùng nhau giải quyết một số vấn đề liên quan đến môn học. Ngoài ra, giảng viên khuyến khích sinh viên chia sẻ kinh nghiệm trong học tập thông qua những buổi seminar, cùng nhau giải quyết các vấn đề nghiên cứu nhằm giải đáp được kết quả mong muốn.
Đồng nghiệp là thành phần thứ hai ảnh hưởng không nhỏ đến hiệu quả giảng dạy của giảng viên. Trong nghiên cứu này các nhóm nhân tố: đồng nghiệp sẵn sàng giúp đỡ trong công việc, sự cạnh tranh của đồng nghiệp, đồng nghiệp là người đáng tin cậy, đồng nghiệp luôn thân thiện và hòa đồng ảnh hưởng đến hiệu quả giảng dạy. Chính vì thế, việc xây dựng và giữ mối quan hệ tốt đẹp với đồng nghiệp sẽ giúp cho cả giảng viên và đồng nghiệp cùng tiến bộ trong công việc. Để có được mối quan hệ tốt đẹp thầy/cô hãy ủng hộ, động viên, giúp đỡ đồng nghiệp vào những lúc cần thiết. Hãy sẵn lòng giúp đỡ, cởi mở và thân thiện với mọi người không những có thể chia sẻ những kinh nghiệm trong giảng dạy mà còn tạo nên một không khí thoải mái, dễ chịu giúp thầy/cô công tác tốt hơn. Trong công tác giảng dạy, việc học hỏi, chia sẻ kinh nghiệm lẫn nhau trong công việc rất quan trọng. Điều này giúp cho giảng viên nâng cao trình độ, chia sẻ phương pháp giảng dạy hiệu quả, giải quyết các vấn đề khó khăn đặc biệt là đối với giảng viên trẻ mới ra trường chưa có nhiều kinh nghiệm trong giảng dạy. Nêu cao tinh thần hợp tác hơn là ganh đua với đồng nghiệp, thường xuyên chia sẻ những đề nghị, ý kiến, thông tin của mình với mọi người trong đơn vị. Đó chính là biện pháp tốt nhất để thầy/cô và đồng nghiệp có dịp gần gũi và hiểu về cách làm việc của nhau. Do đó, trước khi bắt tay vào công việc, thầy/cô và đồng nghiệp nên thảo luận, đặt ra mục tiêu chung về hướng đi, kết quả cần đạt được.

Trường nên tổ chức thực hiện nhiều lớp bồi dưỡng về phương pháp giảng dạy, kỹ năng thuyết trình, kỹ năng giảng dạy kỹ năng giao tiếp, làm việc nhóm, quản lý thời gian cho cán bộ trường. Đồng thời Trường cần có các hoạt động ngoài công tác giảng dạy như các hoạt động đoàn thể, giao lưu giữa các đơn vị trong và ngoài Trường nhằm tăng tính đoàn kêt, học hỏi, giúp đỡ,... lẫn nhau, cùng nhau phát triển và hoàn thiện bản thân là động lực thúc đẩy giảng viên nhiệt huyết trong công tác giảng dạy. Không có gì thuận lợi hơn là làm việc trong một môi trường thân thiện và đoàn kết, điều đó không những tạo hiệu quả trong giảng dạy mà 
còn khiến chính bản thân thầy/cô yêu mến công việc hơn.

Co sở vật chất: các nhóm nhân tố về phương tiện, thiết bị phục vụ giảng dạy, thư viện có đáp ứng nhu cầu giảng dạy và nơi làm việc có thoải mái hay không ảnh hưởng đến hiệu quả giảng dạy của giảng viên. Trường $\mathrm{H}$ CNTT là đơn vị đào tạo về CNTT nên đòi hỏi cần có các trang thiết bị hiện đại nhằm phục vụ cho công tác nghiên cứu, giảng dạy và học tập được tốt hơn.

Lưong, thưởng và phu cấp: các nhóm nhân tố thu nhập đáp ứng được nhu cầu cuộc sống, Trường chi trả phù hợp với năng lực và cao so với mặt bằng chung, các chính sách phúc lợi đa dạng và thể hiện sự quan tâm chu đáo đến giảng viên là nhóm nhân tố ảnh hưởng đến hiệu quả giảng dạy.

Các nguồn lực tài chính được phân bổ hợp lý và sử dụng có hiệu quả số lượng máy móc, trang thiết bị phục vụ giảng dạy, đề tài nghiên cứu khoa học tăng hàng năm, đáp ứng nhu cầu đào tạo, nghiên cứu khoa học và từng bước nâng cao đời sống cán bộ viên chức.

Trường cần có những chính sách phúc lợi, dịch vụ nhằm phục vụ và đáp ứng cả về vật chất lẫn tinh thần cho đội ngũ giảng viên. Hàng năm, Trường cần tổ chức các chuyến tham quan nghỉ mát cho tất cả cán bộ nhân viên và người thân với mục đích thể hiện sự quan tâm của Nhà trường đến toàn thể cán bộ giảng viên. Đồng thời tạo động lực thúc đẩy mối quan hệ giao lưu, học hỏi, chia sẻ kinh nghiệm, là sân chơi để mọi người gần nhau hơn, hiểu nhau hơn, tăng tính đoàn kết nhằm tạo ra hiệu quả làm việc tốt hơn. Và nếu được Nhà trường cần bổ sung thêm các phúc lợi khác cho cán bộ giảng viên ví dụ như có các chính sách an toàn sức khỏe; quà, tiền thưởng nhân các dịp đặc biệt và lễ Tết trong năm nhằm thể hiện sự quan tâm chu đáo, khích lệ tinh thần làm việc của tất cả cán bộ giảng viên.

\section{Tài liệu tham khảo}

[1] Aaronson, D., Barrow, L. \& Sander, W. (2007). Teachers and student achievement in the Chicago public high schools. Journal of Labor Economics. 25(1). pp. 95-135.

[2] Lockwood, J., and D. McCaffrey. 2009. Exploring student-teacher interactions in longitudinal achievement data. Education Finance and Policy. 4 (4) Pp:439-467.

[3] Rivkin, Steven G., Eric A. Hanushek, and John F. Kain. 2005. "Teachers, schools, and academic achievement." Econometrica 73,no.2 (March): 417-458.

[4] Rockoff, JonahE. 2004. "The Impact of Individual Teachers on Student Achievement: Evidence from Panel Data." American Economic Review, 94(2): 247-252.

[5] Rowan, B., Correnti, R., \& Miller, R. 2002. What large-scale, survey research tells us about teacher effects on student achievement: Insights from the Prospects study of elementary schools. Teachers College Record, 104(8), 1525-1567.

[6] Spector, P. E. 1997. Job satisfaction: Application, assessment, causes, and consequences. Thousand Oaks, CA: Sage.

[7] Luddy N. 2005. Job Satisfaction amongst Employees at a Public Health Institution in the Western Cape, University of Western Cape, South McGraw Hill Irwin. Africa.

[8] Cranny, C. J., Smith, P. C. and Stone, E. F. 1992. Job Satisfaction: How People Feel about their Jobs and How it Affects their Perfonnance. Lexington Books: New York.

[9] Ellickson, M.C., \& Logsdon, K. 2002. Determinants of job satisfaction of municipal government employees. Public Personnel Management, Vol.31(3), 343-358.

[10] Nguyễn Đình Thọ \& Nguyễn Thị Mai Trang. 2009. Nghiên cứu khoa học trong quản trị kinh doanh, NXB Thống kê.

[11] Hoàng Trọng - Chu nguyễn Mộng Ngọc. 2008. Phân tích dữ liệu nghiên cứu với SPSS, Trường ĐH Kinh tế TP.HCM, NXB Hồng Đức.

[12] Nunnally, J. C., \& Bernstein, I. H. 1994. Psychometric theory (3rd ed.). New. York; McGraw-Hill. 


\title{
Factors Affecting Lecturers' Teaching Effectiveness
}

\author{
Nguyen Thi Phuong Thao ${ }^{1}$, Vo Van Kiet ${ }^{2}$ \\ ${ }^{1}$ Ho Chi Minh City University of Information Technology, \\ Vietnam National University, Ho Chi Minh City \\ ${ }^{2}$ Nong Lam University, Ho Chi Minh City
}

\begin{abstract}
Teachers' teaching effectiveness is considered as an important determinant of education quality. Once lecturers are satisfied and love their work, they are willing to strive hard to do a better job, which contributes to the success of an education institution in training qualified human resources for the country. This study determines the factors that affect the teaching effectiveness of lecturers. The results of the actual situation analysis show that such factors as student's feedback and result, colleague, facility, salary, bonus and allowance; all affect the lecturer's teaching effectiveness in their own ways.
\end{abstract}

Keywords: Teaching effectiveness, lecturer, training quality. 